\title{
IEEE 802.15.4-PHY Packet Detection and Transmission System With Differential Encoding For Low Power IoT Networks
}

\author{
Y. Siva Krishna, V. Subrahmanyam, M. A. Zubair, P. Rajalakshmi \\ Department of Electrical Engineering \\ Indian Institute of Technology - Hyderabad, India \\ Email: ee14resch11008,ee14mtech11012,ee14mtech01003,raji@iith.ac.in
}

\begin{abstract}
Synchronization is the first operation in a digital baseband receiver. The accuracy of the synchronizer shapes up the performance of the baseband receiver. Synchronization is broadly divided into Carrier Frequency and Phase Synchronization, Symbol Timing and Frame Synchronization. If number of false detections in frame synchronization are high then large amount of power is wasted for processing unwanted packets. In this paper, we are proposing a packet detection and transmission system with differential encoding for low power IoT networks which reduces the number of false packet detections compared to existing preamble detection techniques. The proposed frame synchronization method decides packet is valid or not by cross correlating received packet with fixed preamble sequence and determine its boundaries. The proposed system is analyzed with smart metering power data and corresponding probabilities of packet missing and false detection, power consumption and bit error rate are analyzed. At $3 \mathrm{~dB}$ SNR the number of false detections are reduced by 170 compared to conventional correlation method thereby saving $15.8 \%$ of processing power at receiver.
\end{abstract}

Index Terms-ZigBee, Packet Detection, Differential Encoding, Correlation, Bit Error Rate.

\section{INTRODUCTION}

With the ever increasing wireless traffic, the nodes in an IoT scenario faces a challenge of accurately detecting a packet. Erroneous packet detection can be classified as missing a packet and falsely detecting a packet. The former reduces the throughput and increases the latency in the system. The latter consumes receiver's processing power without giving any valid information. In this paper, we are proposing IEEE 802.15.4PHY packet detection and transmission system with differential encoding which reduces the number of false detections thereby reducing processing power at receiver.

Several frame synchronization and packet detection techniques have been proposed for digital baseband receivers. Correlation of the incoming packet with the known preamble sequence is the most widely used method. In [1], author proved that an optimum detection can be achieved by maximizing the sum of correlation and correction term. In [2], authors rigorously analyzed various techniques and derived that high SNR rule gives nearly optimum detection and are easily implemented. These techniques, however works perfectly for continuous transmission with periodically inserted preamble. But, in IoT scenario, the transmission is asynchronous where

\begin{tabular}{|c|c|c|c|c|}
\hline $\begin{array}{c}\text { Preamble } \\
32 \text { bits }\end{array}$ & $\begin{array}{c}\text { SFD } \\
8 \text { bits }\end{array}$ & $\begin{array}{c}\text { Frame length } \\
7 \text { bits }\end{array}$ & $\begin{array}{c}\text { Reserved bit } \\
1 \text { bit }\end{array}$ & $\begin{array}{c}\text { PSDU } \\
(0-127) \text { bytes }\end{array}$ \\
\hline & \multicolumn{2}{|c|}{ Physical layer Header } & PHY Payload \\
\hline
\end{tabular}

Fig. 1: Physical Layer Packet Structure

sensor node transmits data at random intervals. An Optimum detection rule, an approximation of Maximum Likelihood (ML) rule for asynchronous transmission is derived in [3] for a packet where a fixed bit sequence precedes the random data. IEEE 802.15.4-PHY packet has a fixed 32 bit preamble sequence of all zeros followed by physical header and payload as shown in Fig. 1. A low power ZigBee baseband processor is proposed in [4] where frame synchronization uses a modified delay-and-correlation method. In [5], authors proposed a differential preamble detection where the correlation is applied to a differential encoded data and analyzed the probabilities of missing a preamble and falsely detecting a preamble on a BPSK modulated signals.

In this paper, we are proposing preamble detection with differential encoding technique for low power IoT networks which are using IEEE 802.15.4-PHY radio with Orthogonal Quadrature Phase Shift Keying (OQPSK) modulation. We proposed an optimum threshold for the correlation peak value based on the experimental results of probabilities of missing and falsely detecting a packet. The number of false detections are reduced by using differentially encoding the data packets before transmitting which reduces the processing power consumption at receiver. The Bit Error Rate (BER) comparisons of both proposed and existing packet detection techniques is also analyzed.

The rest of this paper is structured as follows. Section II briefly describes the proposed system architecture for low power IoT networks. Section III discusses the preamble based packet detection with differential encoding. The performance analysis of proposed architecture is presented in Section IV followed by conclusion in Section V. 


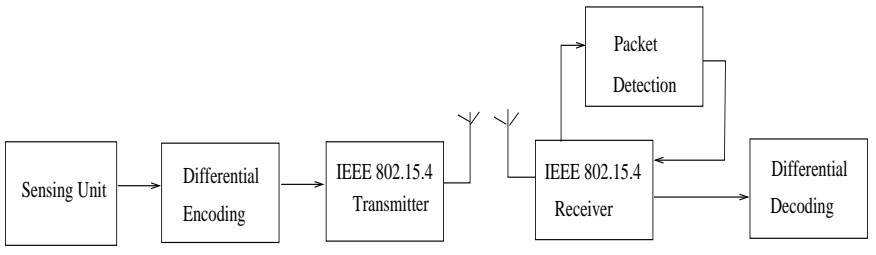

Fig. 2: Proposed Low Power Packet Detection and Transmission System Architecture

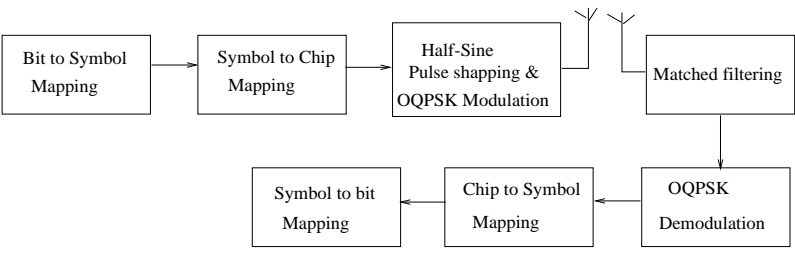

Fig. 3: Block Level Architecture of ZigBee Transmission System

\section{PROPOSED LOW POWER SYSTEM ARCHITECTURE}

The holistic view of proposed low power packet detection and transmission system architecture is shown in Fig. 2. Sensor data is differentially encoded and transmitted by using differential encoder and IEEE 802.15.4 transmitter respectively. Output of packet detection block used as enable signal for receiver. The detailed description of differential encoding and packet detection is presented in next section. The proposed architecture can be applicable to any kind of IoT applications like health care, smart metering, smart home, environmental monitoring etc.. For simulation purpose, we analysed the proposed system with power data collected at IITH by using continuous power monitoring unit. The transmission packet at physical layer is made of physical layer header and data payload from the upper layers. Every packet is differentially encoded before transmitting by using differential encoder. The false packet detections are reduced due to differential encoding which reducing the power consumption at the receiver.

The block level architecture of ZigBee transmission system is as shown in Fig. 3. The maximum input data rate supported by this ZigBee radio is $250 \mathrm{Kbps}$. It converts every four bits as one symbol by using bit to symbol mapping and symbol to chip mapping uses Direct Sequence Spread Spectrum (DSSS) spreading to spread every four bit symbol into a 32 bit chip sequence which increasing data rate to $2 \mathrm{Mbps}$. So, both Inphase and Quadrature-phase (I and Q) components are having $1 \mathrm{Mbps}$ data rate each. The spreading sequences used for IEEE 802.15.4 are presented in [6]. Half Sine (HS) pulse shaping with OQPSK modulation is used to band limit the transmitting pulse and modulate to required band of frequency for transmission.

The received packet at ZigBee receiver under gone filtering to improve signal strength and then demodulated by OQPSK demodulation. Then chip to symbol mapping down converts the resulting $2 \mathrm{Mbps}$ data sequence into four bit

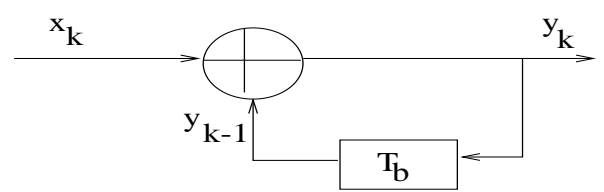

Fig. 4: Differential Encoding

symbols. Symbol to bit mapping used to convert every four bit symbol into bit sequence. The detailed description about IEEE 802.15.4-PHY radio is discussed in [6]. The ZigBee receiver decides whether received packet is to be processed or discarded based on the feedback from the packet detection block. If feedback is binary 1 then ZigBee receiver process the packet else if feedback is binary 0 then it discards the packet without processing.

Packet detection block collects packet from ZigBee receiver and process the packet to find whether it is valid ZigBee packet or not. If it finds that the packet is valid then it sends binary 1 as feedback to receiver else it sends binary 0 as feedback. If packet is valid then data sent by ZigBee receiver is differentially decoded and transmitted to upper layers.

\section{DIFFERENTIAL ENCODING BASED PACKET DETECTION}

The proposed system aims at low power consumption by differentially encoding every packet before transmitting. The added advantage by differential encoding is number of false detections are reduced compared to other transmission systems which are not having encoding method. The differential encoding is done by following the equation (1) which is also shown in Fig. 4. The differentially encoded packet is transmitted using IEEE 802.15.4 radio. At receiver packet is recovered by using ZigBee receiver and transmitted to packet detection block to decide whether it is a valid packet or not.

$$
y_{k}=x_{k} \oplus y_{k-1}
$$

The preamble sequence present in the received packet is correlated with fixed reference sequence to identify it is a valid packet or not.Fig. 5clearly explains procedure involved in the packet detection in proposed architecture. The received packet is cross correlated by taking 32 bits at a time with reference data which is a fixed preamble sequence. In every correlation, the peak value of correlation coefficients is compared with predefined threshold value. If the peak value of correlation is greater than threshold then it decides that packet is valid ZigBee packet and sends binary 1 as feedback to receiver. If the peak value is less than threshold then it decides as invalid packet and sends binary 0 as feedback to receiver.

Correlation is performed by considering a 32 bit window, once the decision is completed window is shifted by one bit and the process is repeated until all the bits of received packet are correlated. As all the packets are transmitted through a noisy channel exact detection of every valid packet may not possible even an optimum threshold value is used and 


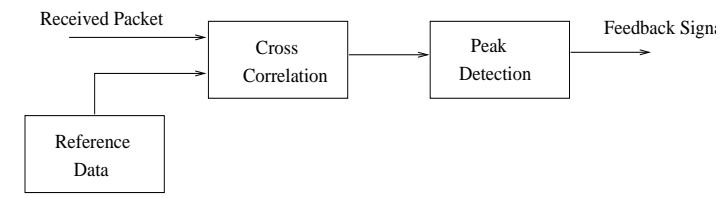

Fig. 5: Block Diagram of Packet Detection Procedure

there may be correlation peak values at other than preamble sequence which are greater than threshold leading to a false packet detection. All these these cases of valid detection, packet missing and false packet detection are considered in our simulations and corresponding results are presented in section IV.

To identify whether the detection is valid or false detection, the last bit time index values of window are stored whenever the correlation peak value is greater than threshold. If the time index value is equal to that of last bit of preamble sequence then it is identified as valid packet detection otherwise it is false detection. If there is no peak detection at the preamble last bit time index then packet is missing. we calculated probabilities of false detection and packet missing and these two are considered as key performance metrics in our simulations. The packet missing probabilities of proposed architecture are almost same as that of correlation method which means the packet missing probabilities are not effected by differential encoding. The false detection probabilities of proposed architecture are reduced compared to correlation method which reduces unwanted packet processing at receiver thereby reducing power consumption. The packet missing probability $\left(P_{m}\right)$ and false detection probability $\left(P_{f}\right)$ are calculated by using equation (2).

$$
P_{m}=\frac{N_{m}}{N_{t}}, P_{f}=\frac{N_{f}}{N_{d}}
$$

Where $N_{m}$ represents number of packets missed, $N_{t}$ is total number of packets transmitted, $N_{f}$ is number false detections and $N_{d}$ represents total number of detections.

\section{Performance analysis of Proposed ARCHITECTURE}

The proposed packet detection and transmission system is analyzed by using the smart metering power data collected at our organization using power monitoring unit. For simulation, we considered packet length as 96 bits and optimum threshold as 28 . All the simulations are performed for 1000 packets with optimum threshold.

\section{A. Comparison of packet missing and false detection proba- bilities}

Packet missing and false detection probabilities are important parameters to be analyzed for proposed packet detection method. By transmitting differentially encoded data there is no change in the packet missing probabilities compared to correlation method without encoding. Fig. 6 reflects the

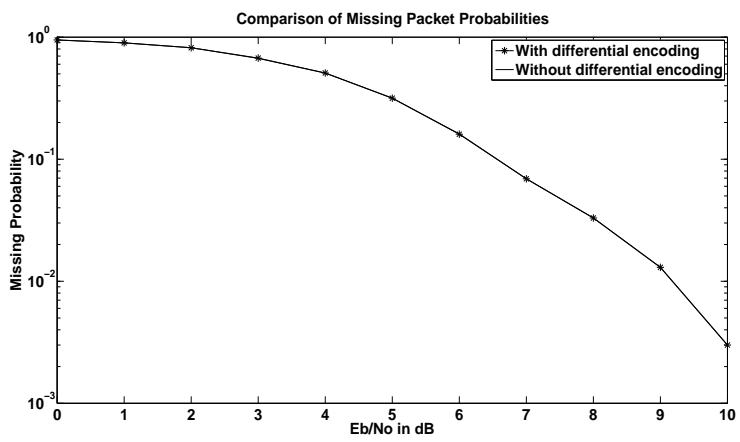

Fig. 6: Comparison of packet missing probabilities for threshold of 28

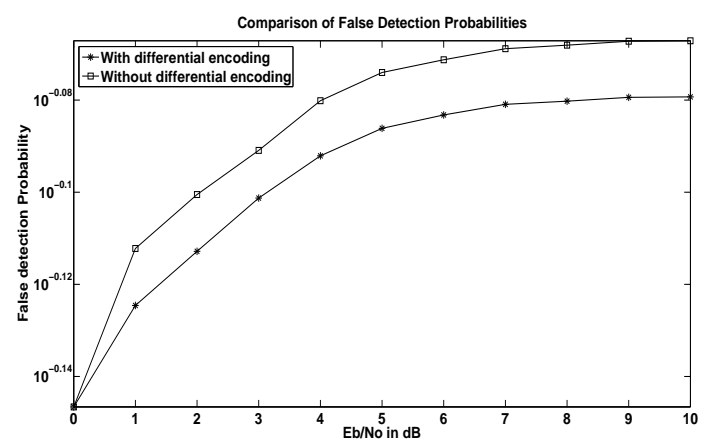

Fig. 7: Comparison of false detection probabilities for threshold of 28

comparison of packet missing probabilities of proposed architecture with that of correlation method. Differential encoding reduces long runs of zeros in transmitting data which reduces the peak values greater than threshold in packets. The false detection probabilities are increasing with SNR for a fixed threshold. The false detection probabilities comparison with respect to SNR for optimum threshold of 28 is shown in 8 . These false detections are further decreased by increasing the threshold value. But, if threshold is increased then packet missing probability is increasing. So, optimum threshold is calculated for which both probabilities are as low as possible. The comparison between packet missing and false detection probabilities for different values of thresholds for a fixed SNR of $0 \mathrm{~dB}$ is shown in Fig. 8. For finding optimum threshold, the average value of point of intersections of both probability curves for different SNRs is calculated. The point of intersections for different SNRs is shown in TABLE I. The ideal value of threshold is 32 and optimum value calculated is 28 .

\section{B. Power consumption analysis}

The power consumption analysis is done by calculating power required for processing one ZigBee packet by assuming $3.3 \mathrm{~V}$ voltage and $17 \mathrm{~mA}$ current consumption in $\mathrm{ON}$ state of 


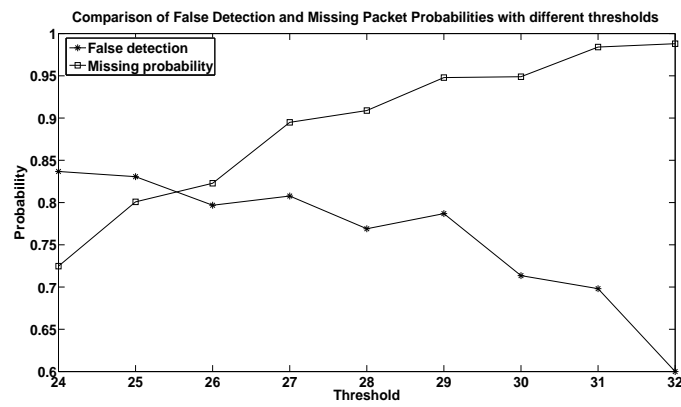

Fig. 8: Packet missing and false detection probabilities comparison for SNR $0 \mathrm{~dB}$

TABLE I: Optimum threshold calculation

\begin{tabular}{|c|c|}
\hline SNR in dB & Point of intersection \\
\hline 0 & 25.5 \\
1 & 27.3 \\
2 & 29.1 \\
3 & 30.8 \\
\hline
\end{tabular}

IEEE 802.15.4-PHY [7]. The equation (3) represents the power consumption for single ZigBee packet.

$$
P_{\text {con }}=\frac{V * I * L}{R}
$$

Where $\mathrm{L}$ is length of packet and $\mathrm{R}$ is maximum transmission rate of ZigBee. The power consumption for false detections for different values of SNRs are compared for both methods of packet detections and presented in Fig. 9 for threshold of 28 . From the Fig. 9, it is clear that at low values of SNR the amount of power saving is very low but if SNR is increasing amount of power saving is also increasing. At SNR $3 \mathrm{~dB}$, the number of false detections are reduced by 170 compared to correlation method. The processing power is saved by 15.8 $\%$ at receiver by using differential encoding method for 1000 packets.

\section{Bit error rate analysis}

The BER analysis for proposed differential encoding based packet detection is almost same as that of packet detection

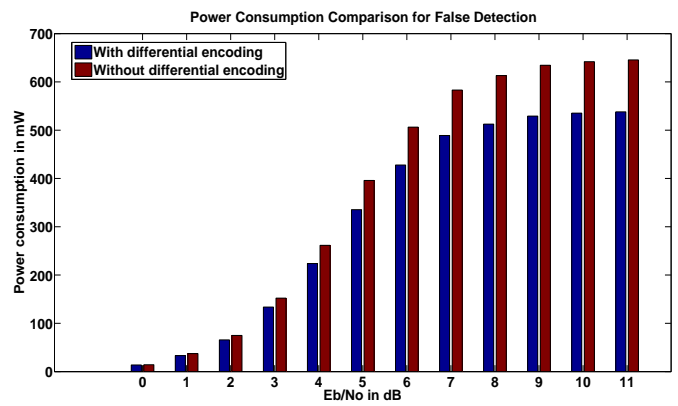

Fig. 9: Power consumption comparison for threshold of 28

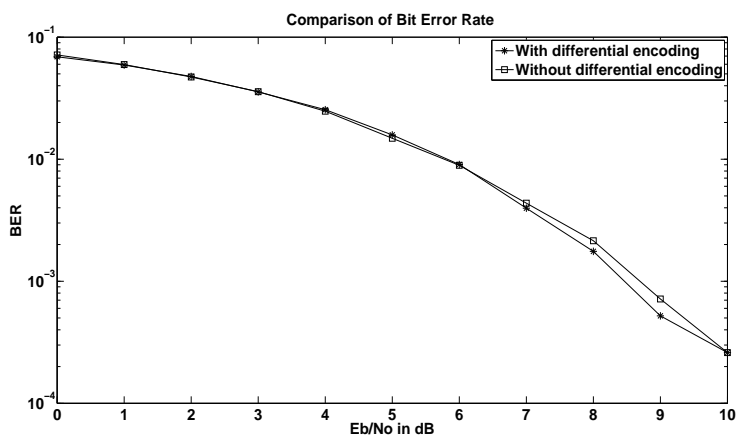

Fig. 10: BER comparison for threshold of 28

method without encoding. Fig. 10 shows the BER comparison of both packet detection methods for different values of SNRs. At From Fig. 10 one can observe that, at low SNRs BER performance is same in both methods and at high SNRs proposed method is performing slightly better than existing method.

\section{CONClusion}

In this paper, we proposed IEEE 802.15.4-PHY packet detection and transmission system with differential encoding for low power IoT applications. The proposed system has less number of false detections compared to correlation based preamble detection method. The performance of proposed architecture is analyzed with smart metering power data and an optimum threshold is selected so that both packet missing and falsely detecting probabilities are as low as possible. The power consumption, BER, packet missing and falsely detecting packet probabilities for optimum threshold are compared with correlation method. The percentage of power saving is $15.8 \%$ in proposed architecture compared to correlation method.

\section{REFERENCES}

[1] Massey, J.L., "Optimum Frame Synchronization," Communications, IEEE Transactions on, vol.20, no.2, pp.115,119, Apr 1972.

[2] Gee Lui; Tan, H.H., "Frame Synchronization for Gaussian Channels," Communications, IEEE Transactions on , vol.35, no.8, pp.818,829, Aug 1987.

[3] Mehlan, R.; Meyr, H., "Optimum frame synchronization for asynchronous packet transmission," Communications, 1993. ICC '93 Geneva. Technical Program, Conference Record, IEEE International Conference on, vol.2, no., pp.826,830 vol.2, 23-26 May 1993.

[4] Kai-Hsin Chen; Hsi-Pin Ma, "A low power ZigBee baseband processor," SoC Design Conference, 2008. ISOCC '08. International, vol.01, no., pp.I-40,I-43, 24-25 Nov. 2008.

[5] Nagaraj, S.; Khan, S.; Schlegel, C.; Burnashev, M.V., "Differential preamble detection in packet-based wireless networks," Wireless Communications, IEEE Transactions on, vol.8, no.2, pp.599,607, Feb. 2009.

[6] Mantri, M.B.; Velagapudi, P.; Eravatri, B.C.; Mani, V.V., "Performance analysis of $2.4 \mathrm{GHz}$ IEEE $802.15 .4 \mathrm{PHY}$ under various fading channels," Emerging Trends in Communication, Control, Signal Processing \& Computing Applications (C2SPCA), 2013 International Conference on , pp.1,4, 10-11 Oct. 2013.

[7] Courtay, A.; Pegatoquet, A.; Auguin, M.; Chabaane, C., "Wireless Sensor Network node global energy consumption modeling," Design and Architectures for Signal and Image Processing (DASIP), 2010 Conference on , pp.54,61, 26-28 Oct. 2010 . 